\title{
Rank generating functions as weakly holomorphic modular forms
}

by

Scott Ahlgren (Urbana, IL) and Stephanie Treneer (Hanover, NH)

Dedicated to Professor Wolfgang Schmidt on the occasion of his seventy-fifth birthday

1. Introduction and statement of results. Recent works have illustrated that the Fourier coefficients of harmonic weak Maass forms of weight $1 / 2$ contain a wealth of number-theoretic and combinatorial information. After these works, it is known that many enigmatic $q$-series (the "mock theta functions" of Ramanujan, and certain rank-generating functions from the theory of partitions, for example) arise naturally as the "holomorphic parts" of such forms. See, for example, Bringmann and Ono [5, 6], Bringmann, Ono, and Rhoades [7], Zwegers [19], Bringmann and Lovejoy [4], Lovejoy and Osburn [12], or see the survey paper [13] for an overview. As another striking example, Bruinier and Ono [9] show that the coefficients of the holomorphic parts of weight $1 / 2$ Maass forms determine the fields of definition of certain Heegner divisors in the Jacobians of modular curves, which in turn determine the vanishing or non-vanishing of derivatives of modular $L$-functions.

Let $p(n)$ denote the number of partitions of $n$, i.e. the number of ways to express $n$ as the sum of a non-increasing sequence of positive integers. The rank of a partition is its largest part minus the number of its parts. Let $0 \leq$ $r<t$ be integers. We denote by $N(r, t, n)$ the number of partitions of $n$ with rank $r \bmod t$. In 1944 Dyson [10] conjectured that the rank functions with modulus 5 and 7 provided a combinatorial explanation for the Ramanujan congruences with these moduli. Dyson's conjectures were proved by Atkin

2000 Mathematics Subject Classification: Primary 11F37; Secondary 11P82.

Key words and phrases: partition function, rank generating function, Maass form, weakly holomorphic modular form.

The first author thanks the National Science Foundation for its support through grant DMS 01-34577. 
and Swinnerton-Dyer [3] in 1954 using the theory of modular functions. In particular, Atkin and Swinnerton-Dyer showed that for all $n$ and all $r$ we have

$$
\begin{aligned}
& N(r, 5,5 n+4)-\frac{1}{5} p(5 n+4)=0, \\
& N(r, 7,7 n+5)-\frac{1}{7} p(7 n+5)=0 .
\end{aligned}
$$

Additionally, Atkin and Swinnerton-Dyer produced many identities for rank generating functions with modulus 5 and 7 (this work was continued by Atkin and Hussain [2] for modulus 11). These identities express such a generating function as a function $h(q)$ which is (up to cosmetic changes) a modular form of weight $1 / 2$ on some congruence subgroup $\Gamma_{1}(N)$, with poles supported at the cusps.

Recent work of Bringmann, Ono, and Rhoades (see Theorem 1.1 of [7]) shows that many of the identities of Atkin and Swinnerton-Dyer represent particular cases of infinite families of identities which express rank generating functions as weakly holomorphic modular forms. These results depend on the equality of the non-holomorphic parts of different weak Maass forms.

In this paper we obtain further infinite families of generating functions involving ranks and partition functions, each member of which is a weakly holomorphic modular form of weight $1 / 2$ on some congruence subgroup $\Gamma_{1}(N)$. When $t=5,7$, and 11, many of the identities of [3] and [2] give examples of these results (we stress that the present work does not identify the particular weakly holomorphic modular form involved, but only the space in which it may be found). To obtain the results, we begin with a family of weak Maass forms (obtained by Bringmann and Ono) whose holomorphic parts are generating functions involving ranks and partitions. We compute the non-holomorphic parts of these forms explicitly, and the results follow from linear relations among them.

Before stating the results, we give two typical examples of the identities of [3]. Define

$$
\eta(z):=q^{1 / 24} \prod_{n=1}^{\infty}\left(1-q^{n}\right), \quad q:=e^{2 \pi i z} .
$$

Let $P(t):=\{t\}^{2}-\{t\}+1 / 6$, and for integers $r$, $t$ with $0<r<t$, define

$$
\eta_{r, t}(z):=q^{t \cdot P(r / t) / 2} \prod_{\substack{n>0 \\ n \equiv \pm r(\bmod t)}}\left(1-q^{n}\right) .
$$

The modular transformation properties of the $\eta_{r, t}(z)$ are well-understood (see, for example, Chapter VIII of the book [15] of Schoeneberg or the paper [14] of Robins). The following identities are equivalent to (6.12) and 
(6.31) of [3], respectively:

$$
\begin{array}{r}
\sum_{n \equiv 4(\bmod 5)}\left(N\left(0,5, \frac{n+1}{24}\right)+2 N\left(1,5, \frac{n+1}{24}\right)-3 N\left(2,5, \frac{n+1}{24}\right)\right) q^{n} \\
=\frac{\eta(600 z) \eta_{10,25}(24 z)}{\left(\eta_{5,25}(24 z)\right)^{2}} \\
\sum_{n \equiv 5(\bmod 7)}\left(N\left(1,7, \frac{n+1}{24}\right)+N\left(2,7, \frac{n+1}{24}\right)\right. \\
\left.=2 N\left(3,7, \frac{n+1}{24}\right)\right) q^{n} \\
=\frac{\eta(1176 z) \eta_{14,49}(24 z)}{\eta_{7,49}(24 z) \eta_{21,49}(24 z)}
\end{array}
$$

Using the criteria of [14] we see that the right sides of (1.3) and (1.4) are weakly holomorphic modular forms of weight $1 / 2$ on $\Gamma_{1}(14400)$ and $\Gamma_{1}(28224)$, respectively. The results below show that a similar phenomenon holds for any odd $t \geq 3$.

To state the results requires some notation. Suppose throughout that $t$ is an odd positive integer, and set

$$
t^{\prime}:=\operatorname{gcd}(t, 3)
$$

Define

$$
\ell_{t}:=\frac{24 t^{2}}{t^{\prime}}, \quad \tilde{\ell}_{t}:=\frac{\ell_{t}}{24}, \quad f_{t}:=\frac{2 t}{t^{\prime}} .
$$

By a weakly holomorphic modular form of weight $k / 2 \in \frac{1}{2} \mathbb{Z}$ on the congruence subgroup $\Gamma_{1}(N)$ we mean a holomorphic function $f: \mathbb{H} \rightarrow \mathbb{C}$ which transforms with the usual automorphy factor in weight $k / 2$ (in the sense of Shimura [16]) under $\Gamma_{1}(N)$. The poles (if any) of such a modular form are supported at the cusps.

In the cases when $t$ is a prime with $\left(\frac{1-24 n}{t}\right)=-1$, the first result can be found in [7].

THEOREM 1.1.

(1) Let $t \geq 3$ be odd and suppose that $0 \leq r<t$. Then

$$
\sum_{n \neq \equiv-(1 \pm 2 r)^{2}(\bmod t)}\left(N\left(r, t, \frac{n+1}{24}\right)-\frac{1}{t} p\left(\frac{n+1}{24}\right)\right) q^{n}
$$

is a weakly holomorphic modular form on $\Gamma_{1}\left(6 f_{t}^{2} \ell_{t} t\right)$.

(2) If $r=(t-1) / 2$ or $r=(t+1) / 2$ then

$$
\sum_{n \neq \equiv-4(\bmod t)}\left(N\left(r, t, \frac{n+1}{24}\right)-\frac{1}{t} p\left(\frac{n+1}{24}\right)\right) q^{n}
$$

is a weakly holomorphic modular form on $\Gamma_{1}\left(6 f_{t}^{2} \ell_{t} t\right)$. 
As an immediate corollary to the first part of this theorem, we obtain the following, which is essentially equivalent to Theorem 1.1 of [7] when $t$ is prime (via twisting, one can extract any particular arithmetic progression of exponents from this sum while preserving modularity). This result explains many of the identities of [3] and [2]; in particular, the identities 13-16, 23, $26-29,32-37$, and 42 from Section 6 of the first paper, and the identities $2-7,9-11,13-18,20,21,23-29,31,34-37,39-42,45-48,50,52,53$, and 55 from Section 10 of the second.

COROllary 1.2. Let $t \geq 3$ be odd and suppose that $0 \leq r_{1}, r_{2}<t$. Then

$$
\sum_{\substack{n \neq \equiv-\left(1 \pm 2 r_{1}\right)^{2}(\bmod t) \\ n \neq \equiv-\left(1 \pm 2 r_{2}\right)^{2}(\bmod t)}}\left(N\left(r_{1}, t, \frac{n+1}{24}\right)-N\left(r_{2}, t, \frac{n+1}{24}\right)\right) q^{n}
$$

is a weakly holomorphic modular form on $\Gamma_{1}\left(6 f_{t}^{2} \ell_{t} t\right)$.

A similar statement can be made for the progressions $r \equiv(t \pm 1) / 2$ $(\bmod t)$. The next two results give other infinite families. Together with Theorem 1.1, they can be used to explain (6.12), (6.18), (6.25), (6.31), and (6.43) of [3] (and so in particular examples (1.3) and (1.4) above). Recall that for all $n, r$, and $t$ we have $N(t-r, t, n)=N(r, t, n)$.

Theorem 1.3. Let $t \geq 5$ be odd.

(a) If $0<r<(t-3) / 2$ then

$$
\sum_{\substack{n \neq \equiv-(1-2 r)^{2}(\bmod t) \\ n \neq-(3+2 r)^{2}(\bmod t)}}\left(N\left(r, t, \frac{n+1}{24}\right)+N\left(r+1, t, \frac{n+1}{24}\right)-\frac{2}{t} p\left(\frac{n+1}{24}\right)\right) q^{n}
$$

is a weakly holomorphic modular form on $\Gamma_{1}\left(6 f_{t}^{2} \ell_{t} t\right)$.

(b) The generating function

$$
\sum_{n \neq-16(\bmod t)}\left(N\left(\frac{t-3}{2}, t, \frac{n+1}{24}\right)+N\left(\frac{t-1}{2}, t, \frac{n+1}{24}\right)-\frac{2}{t} p\left(\frac{n+1}{24}\right)\right) q^{n}
$$

is a weakly holomorphic modular form on $\Gamma_{1}\left(6 f_{t}^{2} \ell_{t} t\right)$.

Theorem 1.4. Let $t \geq 3$ be odd. Then

$$
\sum_{n \neq-9(\bmod t)}\left(N\left(0, t, \frac{n+1}{24}\right)+2 N\left(1, t, \frac{n+1}{24}\right)-\frac{3}{t} p\left(\frac{n+1}{24}\right)\right) q^{n}
$$

is a weakly holomorphic modular form on $\Gamma_{1}\left(6 f_{t}^{2} \ell_{t} t\right)$.

It is possible to combine the generating functions given by Theorems 1.1, 1.3 , and 1.4 in various ways to obtain expressions (like those found in [3]) which do not involve $p(n)$. We give one such example here. 
Corollary 1.5. Suppose that $t \geq 3$ is odd and that $0<r<t$. Then

$$
\sum_{\substack{n \neq \equiv-(1 \pm 2 r)^{2}(\bmod t) \\ n \neq \equiv-9(\bmod t)}}\left(N\left(0, t, \frac{n+1}{24}\right)+2 N\left(1, t, \frac{n+1}{24}\right)-3 N\left(r, t, \frac{n+1}{24}\right)\right) q^{n}
$$

is a weakly holomorphic modular form on $\Gamma_{1}\left(6 f_{t}^{2} \ell_{t} t\right)$.

Note that the case $t=5, r=2, n \equiv 4(\bmod 5)$ gives the left side of $(1.3)$ above. The next result (whose proof in this context is quite simple) explains the identities (1.1), (1.2) (in these cases, the weakly holomorphic modular form in question is identically zero). It also explains identities (10.7), (10.18), (10.29), (10.40), and (10.51) of [2].

Theorem 1.6. Suppose that $t \geq 5$ is prime and that $0 \leq r<t$. Then

$$
\sum_{n=1}^{\infty}\left(N\left(r, t, \frac{t n+1}{24}\right)-\frac{1}{t} p\left(\frac{t n+1}{24}\right)\right) q^{n}
$$

is a weakly holomorphic modular form on $\Gamma_{1}\left(6 f_{t}^{2} \ell_{t}\right)$.

We have one further result.

Theorem 1.7. Suppose that $t \geq 3$ is odd, that $m \geq 5$ is prime, and that $0 \leq r<t$. Then

$$
\sum_{m \nmid n}\left(N\left(r, t, \frac{m n+1}{24}\right)-\frac{1}{t} p\left(\frac{m n+1}{24}\right)\right) q^{n}
$$

is a weakly holomorphic modular form on $\Gamma_{1}\left(6 f_{t}^{2} \ell_{t} m^{2}\right)$.

REMARK 1. We recall that the "mock theta conjectures" (see [1], [11], for example) involve identities for rank generating functions similar to those studied here. In the case of these conjectures, the non-holomorphic part of the functions involved is non-trivial.

REMARK 2. For any particular choice of parameters in these results, it would be possible after some work to give an explicit bound on the pole order of the corresponding weight $1 / 2$ weakly holomorphic modular form. Given this bound, one could provide a proof for any of the explicit identities described here by checking that enough terms of the relevant $q$-expansions agree (unless one is careful with the levels involved, the number of terms which one is required to check to verify these identities can quickly become impractical). One could also construct further explicit identities, although the weakly holomorphic modular forms involved would become more and more complicated to describe.

REMARK 3. These results imply that the rank functions in question satisfy many arithmetic properties. For example, the results of the second 
author $[17,18]$ guarantee the existence of infinitely many linear congruences for the coefficients of these functions.

2. Preliminaries. We recall the definition of a harmonic weak Maass form of half-integral weight $k$ (see Section 3 of [8] for details). If we write $z=x+i y$, the hyperbolic Laplacian of weight $k$ is defined by

$$
\Delta_{k}:=-y^{2}\left(\frac{\partial^{2}}{\partial x^{2}}+\frac{\partial^{2}}{\partial y^{2}}\right)+i k y\left(\frac{\partial}{\partial x}+i \frac{\partial}{\partial y}\right) .
$$

Following Shimura [16] we denote by $\mathcal{G}$ the group of pairs $\xi=(\gamma, \varphi(z))$, where $\gamma=\left(\begin{array}{ll}a & b \\ c & d\end{array}\right) \in \mathrm{GL}_{2}^{+}(\mathbb{Q})$ and $\varphi(z)^{2}= \pm \operatorname{det}(\gamma)^{-1 / 2}(c z+d)$. For $\xi \in \mathcal{G}$ and $k \in \mathbb{Z}$, we define

$$
f[\xi]_{k / 2}(z):=f(\xi z) \varphi(z)^{-k} .
$$

Define $\varepsilon_{d}$ by

$$
\varepsilon_{d}:= \begin{cases}1 & \text { if } d \equiv 1(\bmod 4) \\ i & \text { if } d \equiv 3(\bmod 4) .\end{cases}
$$

For $\gamma=\left(\begin{array}{ll}a & b \\ c & d\end{array}\right) \in \Gamma_{0}(4)$, define $j(\gamma, z) \in \mathcal{G}$ by

$$
j(\gamma, z):=\varepsilon_{d}^{-1}\left(\frac{c}{d}\right)(c z+d)^{1 / 2},
$$

and set $\widetilde{\gamma}:=(\gamma, j(\gamma, z)) \in \mathcal{G}$.

Suppose that $\Gamma \subseteq \Gamma_{0}(4)$ is a congruence subgroup and that $k \in \mathbb{Z}$. A harmonic weak Maass form of weight $k / 2$ on $\Gamma$ is a smooth function $f: \mathbb{H} \rightarrow \mathbb{C}$ with the following properties:

(1) $f[\widetilde{\gamma}]_{k / 2}(z)=f(z)$ for all $\gamma \in \Gamma$.

(2) $\Delta_{k / 2} f=0$.

(3) $f(z)$ has at most linear exponential growth at the cusps.

For convenience, we will assume that the "shadow" of $f$ (i.e. the modular form $y^{k / 2} \frac{\overline{\partial f}}{\partial \bar{z}}$ ) vanishes at infinity (for us, the shadow will always be a cusp form). Such $f$ have a Fourier expansion of the form

$$
f(z)=\sum_{n=n_{0}}^{\infty} a(n) q^{n}+\sum_{n=1}^{\infty} b(n) \Gamma(-k+1,4 \pi n y) q^{-n},
$$

where the $a(n)$ and $b(n)$ are complex numbers, and the incomplete Gamma function is defined by

$$
\Gamma(\alpha, x):=\int_{x}^{\infty} e^{-t} t^{\alpha-1} d t .
$$

We refer to the two sums in (2.1) as the holomorphic and non-holomorphic parts of $f$, respectively. If $N$ is a positive integer with $N \equiv 0(\bmod 4)$, then 
we denote by $\widetilde{\mathcal{M}}_{k / 2}(N)$ the $\mathbb{C}$-vector space of such harmonic weak Maass forms of weight $k / 2$ on $\Gamma_{1}(N)$.

If $l$ is a positive integer, then we define the operator $U(l)$ as follows: if $f(z)$ is as given in (2.1), then

$$
f(z) \mid U(l):=\sum a(\ln ) q^{n}+\sum_{n=1}^{\infty} b(\ln ) \Gamma(-k+1,4 \pi n y) q^{-n} .
$$

Lemma 2.1. Suppose that $N$ is a positive integer with $4 \mid N$ and that $l$ is a positive integer. Define $l_{0}:=\prod_{p \mid l} p$ (where the product is over primes $p$ ) and let $l_{1}$ be the conductor of $\mathbb{Q}(\sqrt{l})$. Set $N^{\prime}:=\operatorname{lcm}\left(N, l_{0}, l_{1}\right)$. Then the operator $U(l)$ maps $\widetilde{\mathcal{M}}_{k / 2}(N)$ to $\widetilde{\mathcal{M}}_{k / 2}\left(N^{\prime}\right)$.

Proof. This follows in the same manner as Proposition 1.5 of [16], with suitable modifications.

We next consider the effect of twisting by a Dirichlet character. If $\chi$ is a Dirichlet character and $f(z)$ is as given in (2.1), then we define the twist of $f$ by $\chi$ via the formula

$$
f(z) \otimes \chi:=\sum_{n=n_{0}}^{\infty} \chi(n) a(n) q^{n}+\sum_{n=1}^{\infty} \chi(-n) b(n) \Gamma(-k+1,4 \pi n y) q^{-n} .
$$

Lemma 2.2. Suppose that $N$ is a positive integer with $4 \mid N$, and that $f(z) \in \widetilde{\mathcal{M}}_{k / 2}(N)$. Suppose that $\chi$ is a Dirichlet character modulo $r$. Set $N^{\prime}:=\operatorname{lcm}\left(N r, r^{2}\right)$. Then $f \otimes \chi \in \widetilde{\mathcal{M}}_{k / 2}\left(N^{\prime}\right)$.

Proof. This follows by a standard argument.

3. Rank generating functions as Maass forms. The results rely on linear relations among the non-holomorphic parts of certain Maass forms, which are developed in this section. We begin with the following theorem of Bringmann and Ono (recall the definitions from (1.6)).

Theorem 3.1 ([6, Theorem 1.3]). If $0 \leq r<t$ are integers with $t$ odd, then

$$
\sum_{n=0}^{\infty}\left(N(r, t, n)-\frac{1}{t} p(n)\right) q^{\ell_{t} n-\widetilde{\ell}_{t}}
$$

is the holomorphic part of a weight $1 / 2$ harmonic weak Maass form on $\Gamma_{1}\left(6 f_{t}^{2} \ell_{t}\right)$. Moreover, the non-holomorphic part of this weak Maass form is given by

$$
\frac{i \ell_{t}^{1 / 2}}{t \sqrt{3}} \sum_{\substack{m\left(\bmod f_{t}\right) \\ n \equiv 6 m+1\left(\bmod 6 f_{t}\right)}} A(r, t, m) \cdot \gamma(t, y ; n) q^{-\widetilde{\ell}_{t} n^{2}},
$$


where

$$
A(r, t, m):=(-1)^{m} \sum_{j=1}^{t-1} \zeta_{t}^{-r j} \sin \left(\frac{\pi j}{t}\right) \sin \left(\frac{\pi j(6 m+1)}{t}\right)
$$

and

$$
\gamma(t, y ; n):=\frac{|n|}{n} \cdot \frac{i}{\sqrt{2 \pi \widetilde{\ell}_{t}}} \cdot \Gamma\left(\frac{1}{2}, 4 \pi \widetilde{\ell}_{t} n^{2} y\right)
$$

Define

$$
\alpha:= \begin{cases}1 / \sqrt{3} & \text { if } 3 \mid t, \\ 1 & \text { if } 3 \nmid t .\end{cases}
$$

Recalling (1.5), we see from Theorem 3.1 that

$$
\begin{aligned}
\sum_{n=0}^{\infty}\left(N(r, t, n)-\frac{1}{t} p(n)\right) q^{\left(24 t^{2} n-t^{2}\right) / t^{\prime}} & \\
+i \alpha \sqrt{8} & \sum_{\substack{m\left(\bmod 2 t / t^{\prime}\right) \\
n \equiv 6 m+1\left(\bmod 12 t / t^{\prime}\right)}} A(r, t, m) \cdot \gamma(t, y ; n) q^{-t^{2} n^{2} / t^{\prime}}
\end{aligned}
$$

is a weight $1 / 2$ harmonic weak Maass form on $\Gamma_{1}\left(6 f_{t}^{2} \ell_{t}\right)$. Applying $U\left(t^{2} / t^{\prime}\right)$ to $(3.4)$, we see that:

$$
\begin{aligned}
\mathcal{R}(r, t ; z):= & \sum_{n=0}^{\infty}\left(N\left(r, t, \frac{n+1}{24}\right)-\frac{1}{t} p\left(\frac{n+1}{24}\right)\right) q^{n} \\
& +\sum_{n \in \mathbb{Z}} \widetilde{A}(r, t, n) \cdot \gamma\left(t, y t^{\prime} / t^{2} ; n\right) q^{-n^{2}}
\end{aligned}
$$

is a weight $1 / 2$ harmonic weak Maass form on $\Gamma_{1}\left(6 f_{t}^{2} \ell_{t}\right)$, where

$$
\widetilde{A}(r, t, n)=i \alpha \sqrt{8} \sum_{\substack{m\left(\bmod 2 t / t^{\prime}\right) \\ n \equiv 6 m+1\left(\bmod 12 t / t^{\prime}\right)}} A(r, t, m) .
$$

Proposition 3.2. Suppose that $t \geq 3$ is odd, let $\widetilde{A}(r, t, n)$ be defined as in (3.6), and let $\alpha$ be defined as in (3.3). Then the following are true:

(a) $\widetilde{A}(0, t, n)= \begin{cases}i \alpha \sqrt{2}(-1)^{(n-1) / 6} \varepsilon t & \text { if } n \equiv \varepsilon(\bmod t) \text { with } \varepsilon \in\{ \pm 1\}, \\ 0 & \text { otherwise. }\end{cases}$

(b) $\widetilde{A}\left(\frac{t-1}{2}, t, n\right)=\widetilde{A}\left(\frac{t+1}{2}, t, n\right)$

$$
=\left\{\begin{array}{l}
\frac{i \alpha}{\sqrt{2}}(-1)^{(n-1) / 6} \varepsilon t \\
\text { if } n \equiv 2 \varepsilon(\bmod t) \text { with } \varepsilon \in\{ \pm 1\}, \\
\text { otherwise. }
\end{array}\right.
$$


(c) If $0 \leq r<t$ and $r \notin\{0,(t-1) / 2,(t+1) / 2\}$, then

$$
\widetilde{A}(r, t, n)=\left\{\begin{array}{l}
\frac{i \alpha}{\sqrt{2}}(-1)^{(n-1) / 6} \text { } \varepsilon t \\
\text { if } n \equiv \varepsilon \pm 2 r(\bmod t) \text { with } \varepsilon \in\{ \pm 1\}, \\
\text { otherwise. }
\end{array}\right.
$$

Proof. Recall (3.6), and consider the case when $3 \nmid t$. Since $n$ is fixed, the double sum in (3.1) contains only one term $A(r, t, m)$, where $m \in$ $\{0, \ldots, 2 t-1\}$ is the unique integer satisfying $m \equiv(n-1) / 6(\bmod 2 t)$. For this $m$ we have

$$
\sin \left(\frac{\pi j(6 m+1)}{t}\right)=\sin \left(\frac{n \pi j}{t}\right)
$$

If $3 \mid t$ then (3.7) also holds. In either case $m$ and $(n-1) / 6$ have the same parity; it follows from (3.2) that

$$
\widetilde{A}(r, t, n)=i \alpha \sqrt{8}(-1)^{(n-1) / 6} \sum_{j=1}^{t-1} \zeta_{t}^{-r j} \sin \left(\frac{\pi j}{t}\right) \sin \left(\frac{n \pi j}{t}\right) .
$$

Using $\sin x=(2 i)^{-1}\left(e^{i x}-e^{-i x}\right)$ and setting $\zeta_{t}:=e^{2 \pi i / t}$, we conclude from (3.8) that

$$
\begin{aligned}
\widetilde{A}(r, t, n)= & i \alpha \sqrt{8}(-1)^{(n-1) / 6} \sum_{j=1}^{t-1} \zeta_{t}^{-r j} \sin \left(\frac{\pi j}{t}\right) \sin \left(\frac{n \pi j}{t}\right) \\
= & -\frac{i \alpha}{\sqrt{2}}(-1)^{(n-1) / 6} \sum_{j=1}^{t-1} \zeta_{t}^{-r j}\left(e^{i \pi j / t}-e^{-i \pi j / t}\right)\left(e^{i \pi n j / t}-e^{-i \pi n j / t}\right) \\
= & -\frac{i \alpha}{\sqrt{2}}(-1)^{(n-1) / 6}\left(\sum_{j=1}^{t-1} \zeta_{t}^{j\left(\frac{n+1-2 r}{2}\right)}-\sum_{j=1}^{t-1} \zeta_{t}^{j\left(\frac{n-1-2 r}{2}\right)}\right. \\
& \left.-\sum_{j=1}^{t-1} \zeta_{t}^{j\left(\frac{-n+1-2 r}{2}\right)}+\sum_{j=1}^{t-1} \zeta_{t}^{j\left(\frac{-n-1-2 r}{2}\right)}\right)
\end{aligned}
$$

(note that each of $\pm n \pm 1-2 r$ is even). Using the fact that

$$
\sum_{j=1}^{t-1} \zeta_{t}^{j\left(\frac{ \pm n \pm 1-2 r}{2}\right)}= \begin{cases}t-1 & \text { if } t \mid( \pm n \pm 1-2 r) / 2, \\ -1 & \text { otherwise, }\end{cases}
$$

the result follows by considering the various possibilities for $r$ and $n \bmod t$.

Proposition 3.3. Let $t \geq 5$ be odd.

(a) Suppose that $0<r<(t-3) / 2$ and that $n \not \equiv \pm(1-2 r), \pm(3+2 r)$ $(\bmod t)$. Then

$$
\widetilde{A}(r, t, n)=-\widetilde{A}(r+1, t, n) .
$$


(b) Suppose that $n \not \equiv \pm 4(\bmod t)$. Then

$$
\widetilde{A}\left(\frac{t-3}{2}, t, n\right)=-\widetilde{A}\left(\frac{t-1}{2}, t, n\right) .
$$

Proof. For part (a), suppose that $0<r<(t-3) / 2$ and that

$$
n \not \equiv \pm(1-2 r), \pm(3+2 r)(\bmod t) .
$$

If $n \not \equiv \pm 1 \pm 2 r(\bmod t)$ and $n \not \equiv \pm 1 \pm 2(r+1)(\bmod t)$, then by Proposition $3.2(\mathrm{c})$ we have

$$
\widetilde{A}(r, t, n)=\widetilde{A}(r+1, t, n)=0,
$$

so the result holds.

Suppose then that one of $n \equiv \pm 1 \pm 2 r(\bmod t)$ or $n \equiv \pm 1 \pm 2(r+1)$ $(\bmod t)$ holds. By $(3.9)$ we conclude that there exists $\varepsilon \in\{ \pm 1\}$ such that $n \equiv \varepsilon(1+2 r)(\bmod t) ;$ it follows that $n \equiv-\varepsilon+2 \varepsilon(r+1)(\bmod t)$. Therefore $\widetilde{A}(r, t, n)=-\widetilde{A}(r+1, t, n)$ by Proposition 3.2(c).

For part (b), we assume that $n \not \equiv \pm 4(\bmod t)$. If $n \not \equiv \pm 2(\bmod t)$, then by Proposition 3.2 we have

$$
\widetilde{A}\left(\frac{t-3}{2}, t, n\right)=\widetilde{A}\left(\frac{t-1}{2}, t, n\right)=0 .
$$

If $n \equiv 2 \varepsilon(\bmod t)$ with $\varepsilon \in\{ \pm 1\}$, then $n \equiv-\varepsilon-2 \varepsilon((t-3) / 2)(\bmod t)$, so the result follows from Proposition 3.2.

Proposition 3.4. Suppose that $t \geq 3$ is odd and that $n \not \equiv \pm 3(\bmod t)$. Then

$$
\widetilde{A}(0, t, n)=-2 \widetilde{A}(1, t, n) .
$$

Proof. Assume that $n \not \equiv \pm 3(\bmod t)$. If $n \not \equiv \pm 1(\bmod t)$, then by parts (a) and (c) of Proposition 3.2 we have

$$
\widetilde{A}(0, t, n)=\widetilde{A}(1, t, n)=0,
$$

so the result holds. If $n \equiv \varepsilon(\bmod t)$ with $\varepsilon \in\{ \pm 1\}$ then the result also follows from Proposition 3.2.

4. Cancellation of the non-holomorphic parts. We prove the results stated in Section 1.

Proof of Theorem 1.1. By (3.5) and Proposition 3.2 the non-holomorphic part of $\mathcal{R}(r, t ; z)$ is supported on terms with exponent $-n^{2}$, where $n \equiv \varepsilon \pm 2 r$ $(\bmod t)$ with $\varepsilon \in\{ \pm 1\}$. It follows from Lemma 2.2 that 


$$
\begin{aligned}
\sum_{n \neq \equiv-(1 \pm 2 r)^{2}(\bmod t)} & \left(N\left(r, t, \frac{n+1}{24}\right)-\frac{1}{t} p\left(\frac{n+1}{24}\right)\right) q^{n} \\
= & \mathcal{R}(r, t ; z)-\frac{1}{\phi(t)} \sum_{\chi(\bmod t)} \bar{\chi}\left(-(1+2 r)^{2}\right) \mathcal{R}(r, t ; z) \otimes \chi \\
& -\frac{1}{\phi(t)} \sum_{\chi(\bmod t)} \bar{\chi}\left(-(1-2 r)^{2}\right) \mathcal{R}(r, t ; z) \otimes \chi
\end{aligned}
$$

is a weakly holomorphic modular form on $\Gamma_{1}\left(6 f_{t}^{2} \ell_{t} t\right)$. The second assertion follows in a similar way from Proposition 3.2 by removing the progression $n \equiv-4(\bmod t)$.

Proof of Theorem 1.3. If $0<r \leq(t-3) / 2$ then by (3.5) we have

$$
\begin{aligned}
& \mathcal{R}(r, t ; z)+\mathcal{R}(r+1, t ; z) \\
& =\sum_{n \geq 0}\left(N\left(r, t, \frac{n+1}{24}\right)+N\left(r+1, t, \frac{n+1}{24}\right)-\frac{2}{t} p\left(\frac{n+1}{24}\right)\right) q^{n} \\
& \quad+\sum_{n \in \mathbb{Z}}(\widetilde{A}(r, t, n)+\widetilde{A}(r+1, t, n)) \cdot \gamma\left(t, y t^{\prime} / t^{2} ; n\right) q^{-n^{2}} .
\end{aligned}
$$

Suppose that $r<(t-3) / 2$. Via twisting, we remove the progressions $n \equiv$ $-(1-2 r)^{2}(\bmod t)$ and $n \equiv-(3+2 r)^{2}(\bmod t)$ from $(4.1)$. By Proposition 3.3, the remaining non-holomorphic part in (4.1) vanishes, and we are left with the weakly holomorphic modular form

$$
\sum_{\substack{n \neq \equiv-(1-2 r)^{2}(\bmod t) \\ n \neq \equiv-(3+2 r)^{2}(\bmod t)}}\left(N\left(r, t, \frac{n+1}{24}\right)+N\left(r+1, t, \frac{n+1}{24}\right)-\frac{2}{t} p\left(\frac{n+1}{24}\right)\right) q^{n} .
$$

If $r=(t-3) / 2$ then we remove the progression $n \equiv-16(\bmod t)$. By part (b) of Proposition 3.3, the remaining sum

$$
\sum_{n \neq \equiv-16(\bmod t)}\left(N\left(\frac{t-3}{2}, t, \frac{n+1}{24}\right)+N\left(\frac{t-1}{2}, t, \frac{n+1}{24}\right)-\frac{2}{t} p\left(\frac{n+1}{24}\right)\right) q^{n}
$$

is a weakly holomorphic modular form.

Proof of Theorem 1.4. From (3.5) we see that

$$
\begin{aligned}
\mathcal{R}(0, t ; z) & +2 \mathcal{R}(1, t ; z) \\
= & \sum_{n \geq 0}\left(N\left(0, t, \frac{n+1}{24}\right)+2 N\left(1, t, \frac{n+1}{24}\right)-\frac{3}{t} p\left(\frac{n+1}{24}\right)\right) q^{n} \\
& +\sum_{n \in \mathbb{Z}}(\widetilde{A}(0, t, n)+2 \widetilde{A}(1, t, n)) \cdot \gamma\left(t, y t^{\prime} / t^{2} ; n\right) q^{-n^{2}} .
\end{aligned}
$$


Via twisting, we remove the progression $n \equiv-9(\bmod t)$ from this sum; by Proposition 3.4, the remaining non-holomorphic part vanishes. Therefore

$$
\sum_{n \neq=-9(\bmod t)}\left(N\left(0, t, \frac{n+1}{24}\right)+2 N\left(1, t, \frac{n+1}{24}\right)-\frac{3}{t} p\left(\frac{n+1}{24}\right)\right) q^{n}
$$

is a weakly holomorphic modular form.

Proof of Theorem 1.6. Suppose that $t \geq 5$ is prime. Applying $U(t)$ to $\mathcal{R}(r, t ; z)$, we obtain

$$
\begin{aligned}
\mathcal{R}(r, t ; z) \mid U(t)= & \sum_{n=0}^{\infty}\left(N\left(r, t, \frac{t n+1}{24}\right)-\frac{1}{t} p\left(\frac{t n+1}{24}\right)\right) q^{n} \\
& +\sum_{n \in \mathbb{Z}} \widetilde{A}(r, t, t n) \cdot \gamma\left(t, y t^{\prime} / t^{3} ; t n\right) q^{-t n^{2}} .
\end{aligned}
$$

If $6 m+1 \equiv t n(\bmod 12 t)$, then $\sin (\pi j(6 m+1) / t)=0$. Using $(3.2)$, we conclude that $A(r, t, m)=0$ for all such $m$; the result follows from (3.6).

Proof of Theorem 1.7. Suppose that $m \geq 5$ is prime. Applying $U(m)$ to $\mathcal{R}(r, t ; z)$, we obtain

$$
\begin{aligned}
\mathcal{R}(r, t ; z) \mid U(m)= & \sum_{n=0}^{\infty}\left(N\left(r, t, \frac{m n+1}{24}\right)-\frac{1}{t} p\left(\frac{m n+1}{24}\right)\right) q^{n} \\
& +\sum_{n \in \mathbb{Z}} \widetilde{A}(r, t, m n) \cdot \gamma\left(t, y t^{\prime} / t^{2} m ; m n\right) q^{-m n^{2}}
\end{aligned}
$$

We have $\mathcal{R}(r, t ; z) \mid U(m) \in \widetilde{M}_{1 / 2}\left(6 f_{t}^{2} \ell_{t} t m\right)$. Theorem 1.7 follows by twisting this form with the trivial character modulo $m$.

\section{References}

[1] G. E. Andrews and F. G. Garvan, Ramanujan's "lost" notebook VI. The mock theta conjectures, Adv. in Math. 73 (1989), 242-255.

[2] A. O. L. Atkin and S. M. Hussain, Some properties of partitions (2), Trans. Amer. Math. Soc. 89 (1958), 184-200.

[3] A. O. L. Atkin and P. Swinnerton-Dyer, Some properties of partitions, Proc. London Math. Soc. (3) 4 (1954), 84-106.

[4] K. Bringmann and J. Lovejoy, Dyson's rank, overpartitions, and weak Maass forms, Int. Math. Res. Not. 2007, no. 19, article ID rnm063.

[5] K. Bringmann and K. Ono, Arithmetic properties of coefficients of half-integral weight Maass-Poincaré series, Math. Ann. 337 (2007), 591-612.

[6] - - - Dyson's ranks and Maass forms, Ann. of Math., to appear.

[7] K. Bringmann, K. Ono, and R. Rhoades, Eulerian series as modular forms, J. Amer. Math. Soc., to appear.

[8] J. H. Bruinier and J. Funke, On two geometric theta lifts, Duke Math. J. 125 (2004), 45-90. 
[9] J. H. Bruinier and K. Ono, Heegner divisors, L-functions, and harmonic weak Maass forms, preprint.

[10] F. Dyson, Some guesses in the theory of partitions, Eureka (Cambridge) 8 (1944), $10-15$.

[11] D. Hickerson, On the seventh order mock theta functions, Invent. Math. 94 (1988), 661-677.

[12] J. Lovejoy and R. Osburn, Rank differences for overpartitions, Quart. J. Math. 59 (2008), 257-273.

[13] K. Ono, Mock theta functions, ranks, and Maass forms, in: Proc. of the Special Year in Number Theory and Combinatorics (2004-2005) at the University of Florida, Springer, to appear.

[14] S. Robins, Generalized Dedekind $\eta$-products, in: The Rademacher Legacy to Mathematics (University Park, PA, 1992), Contemp. Math. 166, Amer. Math. Soc., Providence, RI, 1994, 119-128.

[15] B. Schoeneberg, Elliptic Modular Functions: An Introduction, Springer, New York, 1974.

[16] G. Shimura, On modular forms of half integral weight, Ann. of Math. (2) 97 (1973), 440-481.

[17] S. Treneer, Congruences for the coefficients of weakly holomorphic modular forms, Proc. London Math. Soc. (3) 93 (2006), 304-324.

[18] - Quadratic twists and the coefficients of weakly holomorphic modular forms, J. Ramanujan Math. Soc., to appear.

[19] S. P. Zwegers, Mock theta functions, Ph.D. thesis, Universiteit Utrecht, 2002.

Department of Mathematics

University of Illinois

Urbana, IL 61801, U.S.A.

E-mail: ahlgren@math.uiuc.edu
Department of Mathematics Dartmouth College

Hanover, NH 03755, U.S.A. E-mail: stephanie.treneer@dartmouth.edu

Received on 26.10.2007

and in revised form on 26.3.2008 